\title{
Unconventional Magnetic Ground State in $\mathrm{Yb}_{2} \mathrm{Ti}_{2} \mathrm{O}_{7}$
}

\author{
R. M. D'Ortenzio, ${ }^{1}$ H. A. Dabkowska, ${ }^{2}$ S. R. Dunsiger,${ }^{3}$ B. D. Gaulin,,${ }^{1,2,4}$ \\ M. J. P. Gingras, ${ }^{4,5,6}$ T. Goko, ${ }^{7}$ J. B. Kycia,${ }^{5}$ L. Liu, ${ }^{7}$ T. Medina, ${ }^{1}$ T. J. Munsie, ${ }^{1}$ \\ D. Pomaranski, ${ }^{5}$ K. A. Ross $,{ }^{8}, 9$ Y. J. Uemura, ${ }^{7}$ T. J. Williams, ${ }^{1}$ and G. M. Luke ${ }^{1,2,4, *}$ \\ ${ }^{1}$ Department of Physics and Astronomy, McMaster University, \\ 1280 Main St. W., Hamilton, ON, Canada, L8S $4 M 1$ \\ ${ }^{2}$ Brockhouse Institute for Materials Research, McMaster University, Hamilton, Ontario L8S 4M1, Canada \\ ${ }^{3}$ Physik-Department E21, Technische Universitat Munchen, D-85748 Garching, Germany \\ ${ }^{4}$ Canadian Institute for Advanced Research, Toronto, Ontario, Canada, M5G $1 Z 8$ \\ ${ }^{5}$ Department of Physics and Astronomy, University of Waterloo, Waterloo, ON N2L 3G1, Canada \\ ${ }^{6}$ Perimeter Institute for Theoretical Physics, 31 Caroline North, Waterloo, Ontario, N2L 2Y5, Canada \\ ${ }^{7}$ Department of Physics, Columbia University, New York, New York 10027, USA \\ ${ }^{8}$ Institute for Quantum Matter and Department of Physics and Astronomy, \\ Johns Hopkins University, Baltimore, Maryland 21218, USA \\ ${ }^{9}$ NIST Center for Neutron Research, National Institute of Standards and Technology, Gaithersburg, Maryland 20899, USA
}

(Dated: April 21, 2022)

\begin{abstract}
We report low temperature specific heat and positive muon spin relaxation/rotation ( $\mu \mathrm{SR})$ measurements on both polycrystalline and single-crystal samples of the pyrochlore magnet $\mathrm{Yb}_{2} \mathrm{Ti}_{2} \mathrm{O}_{7}$. This material is believed to possess a spin Hamiltonian able to support a Quantum Spin Ice (QSI) ground state. $\mathrm{Yb}_{2} \mathrm{Ti}_{2} \mathrm{O}_{7}$ displays sample variation in its low temperature heat capacity and, while our two samples exhibit extremes of this variation, our $\mu \mathrm{SR}$ measurements indicate a similar disordered low temperature state down to $16 \mathrm{mK}$ in both. We report little temperature dependence to the muon spin relaxation and no evidence for ferromagnetic order, in contrast to reports by Chang et al. [Nat. Comm. 3, 992 (2012)] and Yasui et al. [J. Phys. Soc. Japan. 72, 11 (2003)]. Transverse field (TF) $\mu$ SR measurements show changes in the temperature dependence of the muon Knight shift that coincide with heat capacity anomalies which, incidentally, prove that the implanted muons are not diffusing in $\mathrm{Yb}_{2} \mathrm{Ti}_{2} \mathrm{O}_{7}$. From these results, we are led to propose that $\mathrm{Yb}_{2} \mathrm{Ti}_{2} \mathrm{O}_{7}$ enters an unconventional ground state below $T_{c} \sim 265 \mathrm{mK}$. As found for all the current leading experimental candidates for a quantum spin liquid state, the precise nature of the state below $T_{c}$ in $\mathrm{Yb}_{2} \mathrm{Ti}_{2} \mathrm{O}_{7}$ remains unknown and, at this time, defined by what it is not as opposed to what it is: lacking simple periodic long-range order or a frozen spin glass state.
\end{abstract}

\section{INTRODUCTION}

The $\mathrm{R}_{2} \mathrm{Ti}_{2} \mathrm{O}_{7}$ rare-earth titanates ( $\mathrm{R}$ is a trivalent magnetic rare earth ion and $\mathrm{Ti}^{4+}$ is non-magnetic) offer outstanding opportunities for the study of geometric magnetic frustration. The $\mathrm{R}$ sites within the cubic pyrochlore structure, with space group $F d \overline{3} m$, form a threedimensional network of corner sharing tetrahedra. This structure has a high propensity towards frustrated magnetic interactions and allows for a wide range of exotic low temperature magnetic states and phenomenologies ${ }^{1}$. For example, classical spin ice pyrochlore materials such as $\mathrm{Dy}_{2} \mathrm{Ti}_{2} \mathrm{O}_{7}$ and $\mathrm{Ho}_{2} \mathrm{Ti}_{2} \mathrm{O}_{7}$, which have been extensively studied and are now relatively well understood $\sqrt{122}$, have been proposed to exhibit monopole-like deconfined quasiparticle excitations ${ }^{3}$.

A new and exciting research direction is that of quantum spin ice, a state in which quantum fluctuations may elevate the classical spin liquid state ${ }^{4}$ of spin ice to a fullblown quantum spin liquid (QSL) 5 10. A possible candidate for a quantum spin ice is $\mathrm{Yb}_{2} \mathrm{Ti}_{2} \mathrm{O}_{7} \sqrt{11}[25$ which does not exhibit the gradual spin freezing and residual lowtemperature entropy of a classical spin ice ${ }^{24 \mid 26}$. The nature of $\mathrm{Yb}_{2} \mathrm{Ti}_{2} \mathrm{O}_{7}$ 's ground state is currently under much debate, with some experiments reporting a ferromagnetic low temperature state $\frac{1423}{}$, while others show a dynamic ground state with no long range order $\frac{13|15| 18 \mid 21}{1}$ and which may be compatible with a QSL state. At the same time, on the theoretical front, the microscopic Hamiltonian for $\mathrm{Yb}_{2} \mathrm{Ti}_{2} \mathrm{O}_{7}$ appears quite well described by a pseudospin$1 / 2$ quantum spin ice mode $\frac{20|24| 25}{2 \text { wich is predicted }}$ within conventiona $\sqrt{20 \mid 27}$ as well as gauge $e^{24}$ mean-field theories to possess a splayed ferromagnetic ground state similar to that recently reported for $\mathrm{Yb}_{2} \mathrm{Sn}_{2} \mathrm{O}_{7}{ }^{\sqrt{28}}$ for the microscopic parameters obtained from inelastic neutron scattering studies of $\mathrm{Yb}_{2} \mathrm{Ti}_{2} \mathrm{O}_{7}$ 20].

Previous zero field (ZF) $\mu \mathrm{SR}^{13116117}$ performed on polycrystalline $\mathrm{Yb}_{2} \mathrm{Ti}_{2} \mathrm{O}_{7}$ showed no evidence of long-range magnetic order below a sharp specific heat transition at $T_{c} \sim 240 \mathrm{mK}$. Rather, an $\mathrm{Yb}^{3+}$ spin fluctuation rate drop of approximately 4 orders of magnitude was observed at $T_{c}$ 1316. Mössbauer absorption spectroscopy measurements by the same group also indicated a first order magnetic transition, but these did not allow for a determination of the ground state.

Different neutron scattering experiments have found evidence for both an ordered ferromagnetic ground state $^{1423}$ and a dynamic magnetic ground state, with only short range (3D) spin correlations 51821 . Rods of magnetic scattering along the $\langle 111\rangle$ directions have

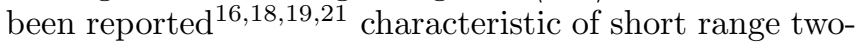


dimensional (2D) correlations above $400 \mathrm{mK}^{21}$. Refs. [21, 18. found that below $400 \mathrm{mK}$, the rods begin to coalesce and build up intensity near the $\boldsymbol{Q}=(1,1,1) \mathrm{nu}-$ clear Bragg peak, interpreted 21 as a cross-over to short range three-dimensional $(3 \mathrm{D})$ magnetic correlations, but clearly lacking long range order. Inelastic neutron scattering at $30 \mathrm{mK}$ in these same studies $\frac{18 \mid 21}{1}$ shows only diffuse quasielastic scattering, and no evidence of sharp spin waves, again consistent with a dynamic disordered ground state. In contrast with Refs. 2118, Chang et al.'s polarized neutron scattering results ${ }^{23}$ show suppression of the $\langle 111\rangle$ scattering rods evolving into magnetic Bragg peaks in a first order manner below $T \sim 210 \mathrm{mK}$, but no concomitant presence of spin waves, as expected for such an ordered state, has so far been reported.

Recent evidence suggests that, like other geometrically frustrated magnets such as $\mathrm{Tb}_{2} \mathrm{Ti}_{2} \mathrm{O}_{7} \frac{\sqrt{29}}{\text {, the ground }}$ state of $\mathrm{Yb}_{2} \mathrm{Ti}_{2} \mathrm{O}_{7}$ may be sensitive to small amounts of quenched disorder and non-stoichiometry at the $\sim 1 \%$ level, with several reports of sample variation of the large heat capacity anomaly near $260 \mathrm{mK}$ and below 21/22/30. Variation of the heat capacity is observed between samples, both poly- and single-crystalline, with the largest heat capacity anomaly appearing at the highest temperature taken as a figure of merit for the sample quality. To date, this anomaly is seen to be sharpest in polycrystalline samples that were prepared by the method outlined in Ref. [22].

Neutron diffraction studies ${ }^{22}$ have shown that a singlecrystal of $\mathrm{Yb}_{2} \mathrm{Ti}_{2} \mathrm{O}_{7}$ grown using the optical floating zone technique is weakly "stuffed", with stoichiometry $\mathrm{Yb}_{2}\left(\mathrm{Ti}_{2-x} \mathrm{Yb}_{x}\right) \mathrm{O}_{7-x / 2}$ where $x \sim 0.046$, or $2.3 \%$ extra $\mathrm{Yb}^{3+}$ ions reside on the nonmagnetic $\mathrm{Ti}^{4+}$ sublattice. Meanwhile, polycrystalline samples prepared using the method in Ref. [22] are seemingly not stuffed $(x \approx 0)$. These defects have been proposed ${ }^{22}$ to contribute to the sample variation in magnetic ground state and specific heat properties.

In this paper, we study two samples of $\mathrm{Yb}_{2} \mathrm{Ti}_{2} \mathrm{O}_{7}$ with very different low temperature heat capacities, shown in Fig. 1. One is a polycrystalline sample with a sharp and large heat capacity anomaly at a relatively high $T_{c} \sim 265 \mathrm{mK}$. The other is a single-crystal sample which displays a broad anomaly at $T_{c} \sim 185 \mathrm{mK}$, with a peak amplitude 20 times smaller than that of the polycrystalline sample. For comparison, we also show the specific heat measurements reported by Chang et al. for the single-crystal used in their neutron study ${ }^{23}$, Dalmas de Réotier et al.'s polycrystalline sample ${ }^{17}$ used in a previous $\mu \mathrm{SR}$ study 13 and Ross et al.'s polycrystal used in Ref. 21]. Based on our specific heat data, we would expect our single-crystal to have a relatively high degree of stuffing as the specific heat peak is pushed down to $\sim 185 \mathrm{mK}$. Our polycrystalline sample shows a sharp peak at the highest temperature so far reported, from which we infer that it should be closer to balanced stoichiometry $(x \approx 0)$. Remarkably, the $\mu \mathrm{SR}$ measurements we report below show little difference between the two samples at low temperatures, and both the single-crystal and our high quality polycrystalline sample are shown to remain disordered and dynamic down to the lowest temperatures considered.

\section{RESULTS}

We prepared polycrystalline pellets of $\mathrm{Yb}_{2} \mathrm{Ti}_{2} \mathrm{O}_{7}$ by mixing stoichiometric quantities of $\mathrm{Yb}_{2} \mathrm{O}_{3}$ and $\mathrm{TiO}_{2}$, isotropically pressing at $60 \mathrm{MPa}$ then sintering at $1200^{\circ} \mathrm{C}$ for 24 hours. We then grew single-crystal $\mathrm{Yb}_{2} \mathrm{Ti}_{2} \mathrm{O}_{7}$ from some of the polycrystalline material using the optical floating zone crystal growth method in 2 ATM of $\mathrm{O}_{2}$ as described in Ref. 31. SQUID measurements of the dcmagnetization above $2 \mathrm{~K}$ confirm Curie-Weiss behavior with overall weak ferromagnetic interactions, $\Theta_{\mathrm{cw}} \approx 0.4$ $\mathrm{K}$, similar to values reported in previous studies ${ }^{11 / 12}$.

We performed specific heat measurements in a ${ }^{3} \mathrm{He} /{ }^{4} \mathrm{He}$ dilution refrigerator at the University of Waterloo, using a combination of the relaxation and sweep methods. Pieces of the single crystal and polycrystalline samples used in the muon spin relaxation measurements described later were sectioned into masses $45.5 \mathrm{mg}$ and $48.0 \mathrm{mg}$, respectively. The samples were thermally connected to a temperature controlled stage through a Manganin wire, with respective thermal conductivities of $6.25 \times 10^{-8} \mathrm{~J} / \mathrm{K} / \mathrm{s}$ and $5.61 \times 10^{-8} \mathrm{~J} / \mathrm{K} / \mathrm{s}$ at $0.48 \mathrm{~K}$. The average step size for the relaxation method was $2-10 \%$ of the nominal temperature, with an equilibration time window at least 3 times the weak link relaxation time constant.



FIG. 1. Low temperature specific heat measurements of $\mathrm{Yb}_{2} \mathrm{Ti}_{2} \mathrm{O}_{7}$ on a semi-logarithmic scale. The red and blue curves represent samples used for this work. Our polycrystalline sample exhibits a sharp transition at $\sim 265 \mathrm{mK}$, and our single-crystal shows a broad peak at $\sim 185 \mathrm{mK}$. The black data is taken from Chang et al.' ${ }^{23}$ single-crystal, and the green is the sample ${ }^{17}$ used for Hodges et al.'s $\mu \mathrm{SR}$ measurements 5 . The orange points are taken from Ross et al. 21 . 


\section{A. Longitudinal Field $\mu$ SR}

Weak longitudinal field (LF) $\mu \mathrm{SR}$ spectra for $T=1$ $\mathrm{K}$ and $16 \mathrm{mK}$ are shown in Fig. 2 for both single-crystal and polycrystalline samples. Calibration spectra using transverse positron counters show that the applied field is $H \leq 0.5 \mathrm{mT}$. We confirmed that the longitudinal relaxation rate did not vary with the strength of the weak applied field (up to at least $\sim 2 \mathrm{mT}$ ) indicating that the small field does not affect the dynamics of the $\mathrm{Yb}^{3+}$ moments, although it does serve to decouple any static nuclear dipole relaxation from any stray muons landing in the cryostat tails or sample holder ${ }^{32}$.
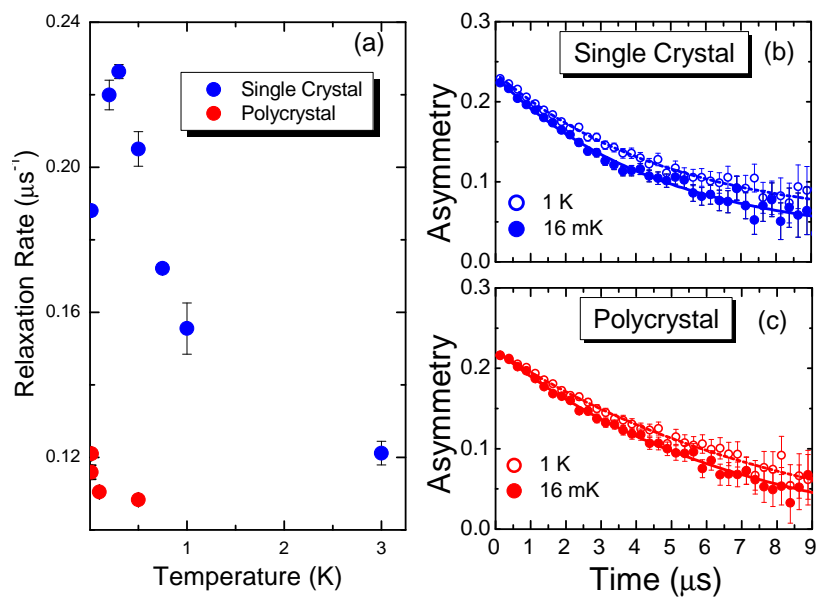

FIG. 2. A comparison of the weak LF (less than $0.5 \mathrm{mT}$ ) data for polycrystalline and single-crystal $\mathrm{Yb}_{2} \mathrm{Ti}_{2} \mathrm{O}_{7}$. Asymmetry spectra (shown in (b) and (c)) were fit with a single exponential decay with a globally fixed asymmetry for all temperatures. Plot (a) shows the temperature dependence of the relaxation rate.

We find that the entire muon polarization signal relaxes with a simple exponential decay characterized by a weak relaxation rate $\lambda(T)$ that is only slightly temperature dependent, as shown in Fig. 2. No oscillatory component or rapid relaxation that would be observed from a state with static moments on the $\mu \mathrm{SR}$ time scale is present down to $16 \mathrm{mK}$ for either sample, noting that the $\mu \mathrm{SR}$ time scale is some six orders of magnitude longer than that relevant to the neutron scattering experiment ${ }^{33}$ finding ferromagnetic order ${ }^{23}$. We can preclude the possibility of a static local field lying solely along the initial muon polarization direction (a situation where no muon precession would be observed) in the polycrystal (where all crystal orientations are present) and in the single crystal (where the cubic symmetry would give 3 equivalent $\langle 111\rangle$ directions, two of which would be perpendicular to the initial muon polarization). In marked contrast to the results presented in Ref. [13], we observe no fast relaxing component of the signal at short time for either of our samples at any temperature, including below the transition (polycrystal) or broad feature (sin- gle crystal) observed in the specific heat measurements in Fig. 1. This behavior allows us to conclude that the $\mathrm{Yb}^{3+}$ spins are in the fast fluctuating (narrowing) regime, with an absence of conventional long range magnetic order or any static internal magnetic fields such as would be present in a spin glass state at low temperature. The relaxation rate does increase somewhat with decreasing temperature, indicating a gradual slowing down of $\mathrm{Yb}^{3+}$ spin fluctuations; however these remain rapidly fluctuating down to $16 \mathrm{mK}$.

\section{B. Transverse Field $\mu$ SR}

To further characterize the low-temperature state of $\mathrm{Yb}_{2} \mathrm{Ti}_{2} \mathrm{O}_{7}$, we performed transverse field (TF) $\mu$ SR measurements to probe the local spin susceptibility, applying $H=50 \mathrm{mT}$ perpendicular to the initial muon spin polarization, which was rotated $90^{\circ}$ relative to the LF measurements. For the single-crystal $\mathrm{Yb}_{2} \mathrm{Ti}_{2} \mathrm{O}_{7}$, the field was applied parallel to [111]. A field of $H=50 \mathrm{mT}$ along [110] is small enough not to induce a magnetically ordered state and likely also for the [111] direction $18 / 21 / 34$. Fig. 3 shows Fourier transforms of time spectra measured at $T=50 \mathrm{mK}$, which exhibit a number of resolved lines for each sample. The positive muon site in materials is determined by electrostatic interactions, with muons in $\mathrm{Yb}_{2} \mathrm{Ti}_{2} \mathrm{O}_{7}$ most likely to reside near $\mathrm{O}^{2-}$ ions ${ }^{32}$. There are two crystallographically inequivalent $\mathrm{O}^{2-}$ ions in the pyrochlore structure ${ }^{1}$, both of which could provide muon sites. The application of an external magnetic field along a specific (in this case a [111]) axis polarizes the $\mathrm{Yb}^{3+}$ moments, making several crystallographically equivalent sites magnetically inequivalent, which therefore increases the multiplicity of muon precession frequencies. The splitting of each line from the frequency corresponding to the applied field reflects the local (anisotropic) susceptibility probed at that particular muon site. The polycrystalline sample exhibits fewer lines, though they are broader in frequency as a result of the powder-averaging of the anisotropic shifts of different crystallographic sites. After examining the Fourier transforms we chose to fit the time spectra to Eq. 1 with $n=3$ (polycrystal) and $n=4$ (single-crystal), fixing the amplitudes of the individual components at all temperatures for each sample to values obtained from simultaneous global fits over a range of temperatures. Typical fits are shown in the insets of Fig. 4 (a) and (b) for the polycrystal and single-crystal samples, respectively.

$$
A(t)=\sum_{i=1}^{n} A_{i} e^{-\sigma_{i}^{2} t^{2}} \cos \left(\omega_{i} t+\varphi_{i}\right)
$$

We show the results of this analysis for the frequencies of each component in Fig. 4. The highest frequency component (labeled in grey) for each sample exhibits no temperature dependence. We ascribe this signal to 


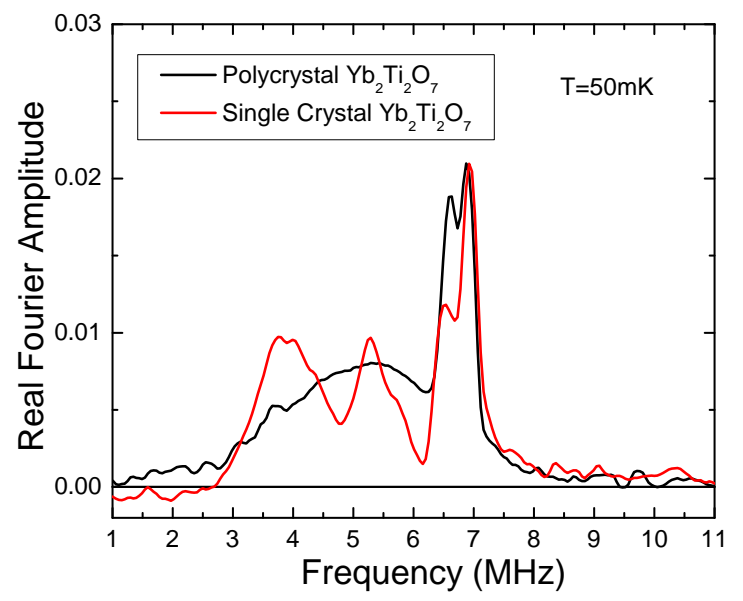

FIG. 3. Fourier Transform of $\mu \mathrm{SR}$ asymmetry spectra in a transverse field of $50 \mathrm{mT}$ at $T=50 \mathrm{mK}$. The single-crystal $\mathrm{Yb}_{2} \mathrm{Ti}_{2} \mathrm{O}_{7}$ shows 4 resolvable frequencies (red) and the polycrystalline $\mathrm{Yb}_{2} \mathrm{Ti}_{2} \mathrm{O}_{7}$ shows three (black). This motivates $n=4$ for the single-crystal and $n=3$ in the polycrystalline $\mathrm{Yb}_{2} \mathrm{Ti}_{2} \mathrm{O}_{7}$ for Eq. (1). The sharp signal at approximately 7 $\mathrm{MHz}$ for both samples reflects the precession of the muons in the Ag cryostat tails.

muons landing in the silver sample holder which provides a useful reference signal for determining the muon Knight shift. The frequencies originating from the samples all show a strong temperature dependence as shown in Fig 4 . We parametrized the Knight shifts of these signals referenced to the silver signal by fitting them to a CurieWeiss temperature dependence in the temperature range $400 \mathrm{mK}<T<3.5 \mathrm{~K}$. We used a common " $\mu \mathrm{SR}$ CurieWeiss temperature", $T_{\mathrm{cW}}^{\mu}$, for each frequency signal from a given sample, but allowed $T_{\mathrm{cW}}^{\mu}$ to vary between samples. The resulting frequencies obtained from these fits of the Knight shifts are indicated by the black solid lines in Fig. 4. for both samples we find $T_{\mathrm{cw}}^{\mu}=-1.3 \pm 0.5 \mathrm{~K}$, which is considerably below the value $\left(\Theta_{\mathrm{cw}}=+0.4 \mathrm{~K}\right)$ we obtain at higher temperatures (above $2 \mathrm{~K}$ ) from dcmagnetization measurements. For both samples we see a large and rather abrupt deviation from this parametrized Curie-Weiss behavior below about $T \approx 0.25 \mathrm{~K}$. This temperature corresponds to the onset of the specific heat phase transition in the polycrystal and is near to that of the single crystal, both indicated by the vertical arrows in Fig. 4. The deviation is most pronounced in the polycrystalline sample where the specific heat transition is also the most pronounced. The change in the temperature dependence of the Knight shift indicates that there is a marked change in either the local spin susceptibility and/or the $\mathrm{Yb}^{3+}$-muon hyperfine coupling, onsetting around the thermodynamic phase transition in $\mathrm{Yb}_{2} \mathrm{Ti}_{2} \mathrm{O}_{7}$ as identified by the specific peak in Fig. 2 .

The similarities of our data between the polycrystalline

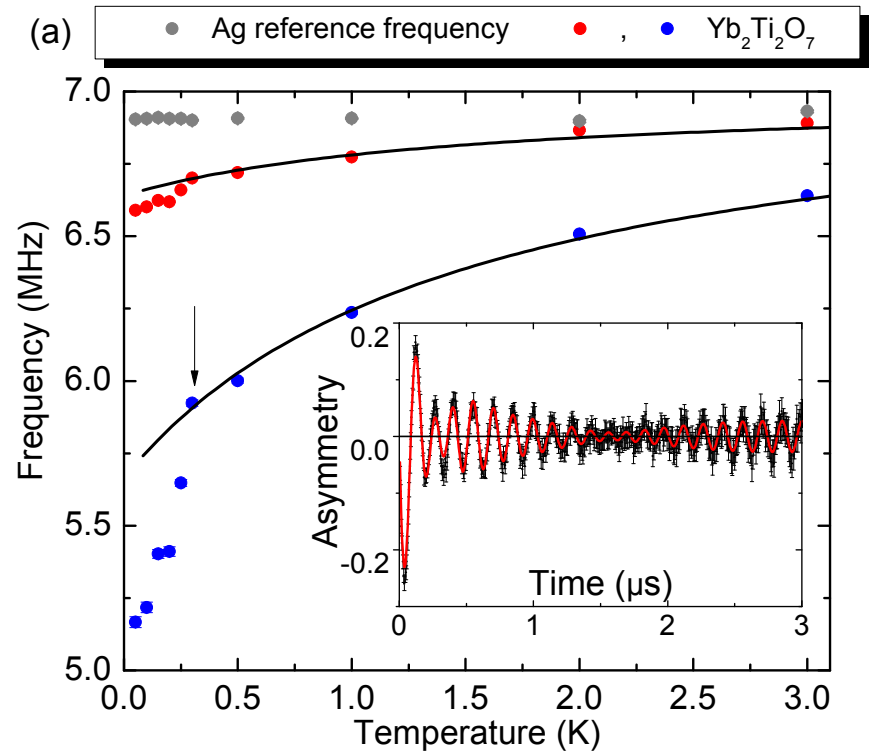

(b) $\bullet$ Ag reference frequency $\bullet \quad, \quad, \quad \mathrm{Yb}_{2} \mathrm{Ti}_{2} \mathrm{O}_{7}$



FIG. 4. The $\mathrm{Yb}^{3+}$ spin susceptibility characterized by TF- $\mu \mathrm{SR}$ measurements of (a) polycrystalline and (b) singlecrystal $\mathrm{Yb}_{2} \mathrm{Ti}_{2} \mathrm{O}_{7}$. For the single-crystal $\mathrm{Yb}_{2} \mathrm{Ti}_{2} \mathrm{O}_{7}$ the initial muon polarization points perpendicular to the $\mathrm{TF}=50 \mathrm{mT}$ field which was applied parallel to [111]. There are two distinct muon signals from the polycrystalline sample, and three from the single-crystal sample. The arrows show the approximate temperature of the onset of the transition observed in the specific heat. The solid black lines represent Curie-Weiss fits, as described in the text. The insets show asymmetry spectra (black) and fit to Eq. 1 (red).

and single-crystal samples is surprising considering their very different specific heat signatures indicating a wide range of stuffing 22 . It is also surprising that we see no evidence for the dramatic first-order-like change in the spin fluctuation rate that was previously reported 13 . This difference is apparent in the raw data of Figs. $2 \mathrm{~b}$ and $2 \mathrm{p}$ and is evidence of a sample dependence between those stud- 
ied by Hodges et al. ${ }^{13 \mid 16}$ and this work which must go beyond the differences in stuffing between our polycrystal and single-crystal samples.

Most importantly, our results suggest that, from a local microscopic point of view, the magnetic ground state is to a large extent insensitive to some aspects of sample variation and that a sharp specific heat transition does not signify magnetic order.

Our weak LF measurements, shown in Fig. 2 exhibit a weakly temperature dependent spin fluctuation rate down to the lowest temperature considered. Many frustrated pyrochlore systems exhibit a substantial, largely temperature independent LF/ZF relaxation 32 35 37 which, although often seen in frustrated or low-dimensional magnetic systems, have not yet been explained $\sqrt{36}$. A recently proposed explanation for these apparent persistent spin dynamics in terms of quantum diffusion of muons, and suggested for $\mathrm{Dy}_{2} \mathrm{Ti}_{2} \mathrm{O}_{7} \frac{37}{\text {, is un- }}$ tenable here (and likely in that case as well as we would expect similar muon diffusion behavior in the two systems). If muons were mobile in $\mathrm{Yb}_{2} \mathrm{Ti}_{2} \mathrm{O}_{7}$, they would most likely move between different magnetically inequivalent sites which would broaden all of the individual TF lines in Fig. 3 into one, which clearly does not occur. A recent ZF- $\mu$ SR study of $\mathrm{Y}_{2} \mathrm{Ti}_{2} \mathrm{O}_{7}$ also concluded that quantum diffusion was absent in that related materia 38 . Furthermore, we can exclude a "muon impurity" effect in which the presence of the muon would locally destroy the magnetic behavior, since our $\mathrm{TF}-\mu \mathrm{SR}$ measurements are clearly sensitive to the transition which strongly affects our measured muon Knight shift (see Fig. 4).

\section{DISCUSSION}

$\mathrm{Yb}_{2} \mathrm{Ti}_{2} \mathrm{O}_{7}$ in the polycrystalline (and stoichiometric) form exhibits a clear thermodynamic phase transition evidenced by specific heat measurements that is accompanied by the marked change in the muon Knight shift in Fig. 4a. On the other hand, the broad anomaly observed in the single crystal heat capacity may be indicative of a crossover, rather than a thermodynamic phase transition, and consistent with the smoother shift seen in Fig. 4b compared to Fig. 4a. We did not perform a detailed search for hysteresis at this transition which precludes us from making a definitive statement regarding its order. The specific heat anomaly in the polycrystal is extremely sharp which could be evidence for a first order transition, but further measurements will be required to confirm this. This leaves the nature of the low temperature state unresolved, although we do know that it involves at least a change of the local spin susceptibility and/or hyperfine coupling, rather than complete magnetic ordering. This behavior reminds that of the moderate heavy fermion system $\mathrm{URu}_{2} \mathrm{Si}_{2}$ which has a thermodynamic phase transition at $17.5 \mathrm{~K}$ to a so-called "hidden-order" state $^{39}$ without magnetic order and whose order parameter has remained unknown for more than 20 years of de- tailed investigation. In the case of $\mathrm{URu}_{2} \mathrm{Si}_{2}$, recent studies $\sqrt{40}$ indicate that the phase transition originates from a change in the hybridization between the localized electronic states associated with the uranium atoms and the conduction band, as it enters the heavy fermion state. As such a mechanism cannot occur in insulating $\mathrm{Yb}_{2} \mathrm{Ti}_{2} \mathrm{O}_{7}$, we must seek another mechanism for its phase transition to a state with an unidentified order parameter.

When discussing "hidden-order", one often refers to the ordering of other multipoles than the magnetic dipole moment ${ }^{41}$, with the electric quadrupolar (E2) being typically the most important one after the magnetic dipolar moment. A transition that would involve the electrical quadrupole moments of $\mathrm{Yb}^{3+}$ at the first order transition in $\mathrm{Yb}_{2} \mathrm{Ti}_{2} \mathrm{O}_{7}$ would seem hard to rationalize given that $\mathrm{Yb}^{3+}$ is a Kramers ion with vanishing time even (e.g. quadrupolar) operator matrix elements projected in its low-energy crystal-field doublet ${ }^{9}$ along with a lowest excited crystal field levels at an energy $\sim 600 \mathrm{~K}$ very large compared to the energy scale of the relevant microscopic $\mathrm{Yb}^{3+}-\mathrm{Yb}^{3+} \mathrm{E} 2$ interactions. That being said, numerous exotic ground states have been proposed $\frac{8110}{}$ for effective spin- $1 / 2$ pyrochlore Kramers systems, such as $\mathrm{Yb}_{2} \mathrm{Ti}_{2} \mathrm{O}_{7}$. These include a $U(1)$ quantum spin liquid (QSL) and a Coulomb ferromagnet in addition to conventional antiferromagnetic and ferromagnetic long-range order. Interestingly, the recent suggestion of a first order transition between a paramagnet and the $U(1) \mathrm{QSL}^{10}$ could be consistent with what we observe in $\mathrm{Yb}_{2} \mathrm{Ti}_{2} \mathrm{O}_{7}$. Another possibility which could be considered is a valence bond solid (VBS). Of these states, the QSL and VBS would have no static magnetic moments, consistent with our results. However, it is unclear whether or how the known Hamiltonian for $\mathrm{Yb}_{2} \mathrm{Ti}_{2} \mathrm{O}_{7} 20124|25| 27$ could give rise to either of these states. Which of these phases or perhaps other more exotic states is realized in $\mathrm{Yb}_{2} \mathrm{Ti}_{2} \mathrm{O}_{7}$ remains to be seen.

The search for materials with a QSL ground state is at the forefront of theoretical and experimental condensed matter research ${ }^{4}$. Notwithstanding the paucity of such systems, one of the fundamental conceptual challenges facing the field is that the identification of a QSL ground state has so far typically been "what it is not" (i.e. lacking static multipolar order) rather than what it is $\$ 442$. Moving beyond this negative assessment of a QSL is one of the central questions being investigated in the field. On the experimental side, one still has to remain cautiously satisfied in obtaining a compelling demonstration of circumstantial evidence for a QSL state. An example is the recently reported gapless fractionalized magnetic (spinons) excitations in the intensively studied $\mathrm{ZnCu}_{3}(\mathrm{OD})_{6} \mathrm{C}_{12}$ Herbertsmithite $\mathrm{Cu}^{2+} S=1 / 2$ kagome antiferromagnet $\underline{43}$.

In our work, we have brought $\mathrm{Yb}_{2} \mathrm{Ti}_{2} \mathrm{O}_{7}$ into the not very crowded arena of compelling QSL candidates by showing, as a first essential step for further progress, that it is an effective $S=1 / 2$ three-dimensional frustrated spin system lacking static dipolar order above $16 \mathrm{mK}$. 
We hope that our work encourages others further systematic experimental and theoretical studies of this most interesting compound.

\section{ACKNOWLEDGMENTS}

Research at McMaster University and University of Waterloo is supported by NSERC while research at
Columbia is supported by the US NSF PIRE (OISE0968226) and DMR-1105961 projects. We appreciate the technical support of Dr. B. Hitti and Dr. A. Dabkowski and the TRIUMF Centre for Molecular and Materials Science. This work is supported in part by the Canada Research Chair program (M.J.P.G., Tier 1) and by the Perimeter Institute for Theoretical Physics through Industry Canada and by the Province of Ontario through the Ministry of Economic Development \& Innovation.
* luke@mcmaster.ca

1 J.S. Gardner, M.J.P. Gingras, and J.E. Greedan. Rev. Mod. Phys. 82, 53-107 (2010).

2 M. J. P. Gingras, Spin Ice. Chapter 12 in Introduction to Frustrated Magnetism, edited by C. Lacroix, P. Mendels, and F. Mila (Springer Series in Solid-State Sciences, Heidelberg, 2011).

3 C. Castelnovo, R. Moessner, and S.L. Sondhi, Nature, 451, 42 (2008).

${ }^{4}$ L. Balents, Nature 464, 199 (2010);

${ }^{5}$ M. Hermele, M. P. A. Fisher, and L. Balents, Phys. Rev. B, 69, 064404 (2004).

${ }^{6}$ H. R. Molavian, M. J. P. Gingras, and B. Canals Phys. Rev. Lett. 98, 157204 (2007).

7 S. Onoda and Y. Tanaka, Phys. Rev. Lett. 105, 047201 (2010).

8 L. Savary and L. Balents, Phys. Rev. Lett. 108, 037202 (2012).

9 SungBin Lee, S. Onoda, and L. Balents, Phys. Rev. B 86, 104412 (2012).

10 L. Savary and L. Balents, Phys. Rev. B 87, 205130 (2013).

11 S. T. Bramwell, M. N. Field, M. J. Harris, and I. P. Parkin, J. Phys. Condens. Matter 12483 (2000).

12 J. A. Hodges, P. Bonville, A. Forget, M. Rams, K. Królas, and G. Dhalenne, J. Phys. Condens. Matter 139301 (2001).

13 J.A. Hodges, P. Bonville, A. Forget, A. Yaouanc, P. Dalmas de Réotier, G. André, M. Rams, K. Królas, C. Ritter, P.C.M. Grubbens, C. T. Kaiser, P. J. C. King, and C. Baines. Phys. Rev. Lett. 88, 077204 (2002).

14 Y. Yasui, M. Soda, S. Iikubo, M. Ito, M. Sato, N. Hamaguchi, T. Matsushita, N. Wada, T. Takeuchi, N. Aso, and K. Kakurai. J. Phys. Soc. Japan. 72, 11 (2003).

15 J. S. Gardner, G. Ehlers, N. Rosov, R. W. Erwin, and C. Petrovic. Phys. Rev. B 70, 180404 (2004).

16 P. Bonville, J. A. Hodges, E. Bertin, J. Ph. Bouchaud, P. Dalmas de Réotier, L.-P. Regnault, H.M. Rønnow, J.-P. Sanchez, S. Sosin and A. Yaouanc. Hyper. Inter. 156/157, 103-111 (2004).

17 P. Dalmas de Réotier, V. Glazkov, C. Martin, A. Yaouanc, P.C.M. Gubbens, S. Sakarya, P. Bonville, A. Amato, C. Baines, and P. J. C. King. Physica B. 374-375, 145-147 (2006).

18 K. A. Ross, J. P. C. Ruff, C. P. Adams, J. S. Gardner, H. A. Dabkowska, Y. Qiu, J. R. D. Copley, and B. D. Gaulin. Phys. Rev. Lett. 103, 227202 (2009).

19 J. D. Thompson, P.A. McClarty, H. M. Rønnow, L. P. Regnault, A. Sorge, and M. J. P. Gingras, Phys. Rev. Lett. 106, 187202 (2011).

${ }^{20}$ K. A. Ross, L. Savary, B. D. Gaulin and L. Balents, Phys.
Rev. X 1, 021002 (2011).

21 K. A. Ross, L. R. Yaraskavitch, M. Laver, J. S. Gardner, J. A. Quilliam, S. Meng, J. B. Kycia, D. K. Singh, Th. Proffen, H. A. Dabkowska, and B. D. Gaulin, Phys. Rev. B 84, 174442 (2011).

${ }^{22}$ K. A. Ross, Th. Proffen, H. A. Dabkowska, J. A. Quilliam, L. R. Yaraskavitch, J. B. Kycia, and B. D. Gaulin. Phys. Rev. B. 86174424 (2012).

${ }^{23}$ L.-J. Chang, S. Onoda, Y. Su, Y.-J. Kao, K.-D. Tsuei, Y. Yasui, K. Kakurai, and M. R. Lees. Nat. Comm. 3, 992 (2012).

24 R. Applegate, N. R. Hayre, R. R. P. Singh, T. Lin, A. G. R. Day, and M. J. P. Gingras, Phys. Rev. Lett. 109, 097205 (2012).

25 N. R. Hayre, K. A. Ross, R. Applegate, T. Lin, R. R. P. Singh, B. D. Gaulin, and M. J. P. Gingras, Phys. Rev. B 87, 184423 (2013).

${ }^{26}$ We note, however, that a recent paper [D. Pomaranski, L. R. Yaraskavitch, S. Meng, K. A. Ross, H. M. L. Noad, H. A. Dabkowska, B. D. Gaulin, and J. B. Kycia, Nature Physics 9, 353 (2013)] finds no residual Pauling entropy at $T \lesssim 500 \mathrm{mK}$ in $\mathrm{Dy}_{2} \mathrm{Ti}_{2} \mathrm{O}_{7}$, unlike all previous measurements on this compound.

27 A. W. C. Wong, Z. Hao, and M. J. P. Gingras, Phys. Rev. B 88, 144402 (2013).

28 A. Yaouanc, P. Dalmas de Réotier, P. Bonville, J. A. Hodges, V. Glazkov, L. Keller, V. Sikolenko, M. Bartkowiak, A. Amato, C. Baines, P. J. C. King, P. C. M. Gubbens, and A. Forget, Phys. Rev. Lett. 110, 127207 (2013).

${ }^{29}$ H. Takatsu, H. Kadowaki, T. J. Sato, J. W. Lynn, Y. Tabata, T. Yamazaki, and K. Matsuhira, J. Phys. Cond. Matt. 24, 052201 (2012).

30 A. Yaouanc, P. Dalmas de Réotier, C. Martin, and V. Glazkov. Phys. Rev. B. 84, 172408 (2011).

31 Springer Handbook of Crystal Growth, Chapter 12. H. A. Dabkowska and A. Dabkowski (Springer, Verlag Berlin Heidelberg) (2010).

32 S. R. Dunsiger, A. A. Aczel, C. Arguello, H. Dabkowska, M.-H. Du, T. Goko, B. Javanparast, T. Lin, F. L. Ning, H. M. L. Noad, D.J. Singh, T. J. Williams, Y. J. Uemura, M. J. P. Gingras, and G. M. Luke. Phys. Rev. Lett. 107, 207207 (2011).

33 P. Dalmas de Réotier, A. Yaouanc, L. Keller, A. Cervellino, B. Roessli, C. Baines, A. Forget, C. Vaju, P. C. M. Grubbens, A. Amato, and P. J. C. King, Phys. Rev. Lett. 96, 127202 (2006).

34 See discussion in Section V.C of Ref. 25.

35 Y. J. Uemura A. Keren, K. Kojima, L. P. Le, G. M. Luke, W. D. Wu, Y. Ajiro, T. Asano, Y. Kuriyama, M. Mekata, 
H. Kikuchi, and K. Kakurai. Phys. Rev. Lett. 73, 3306 (1994).

${ }^{36}$ P. A. McClarty, J. N. Cosman, A. G. Del Maestro, and M. J. P. Gingras, J. Phys. Condens. Matter. 23, 164216 (2011).

37 P. Quémerais, P. A. McClarty, and R. Moessner, Phys. Rev. Lett. 109, 127601 (2012).

38 J. A. Rodriguez, A. Yaouanc, B. Barbara, E. Pomjakushina, P. Quemarais and Z. Salman, Phys. Rev. B. 87, 184427 (2013).

39 J. A. Mydosh abd P. M. Oppeneer. Rev. Mod. Phys. 83, 1301 (2011).
40 A. R. Schmidt, M. H. Hamidian, P. Wahl, F. Meier, A. V. Balatsky, J. D. Garrett, T. J. Williams, G. M. Luke, and J. C. Davis, Nature 465, 570 (2010).

41 P. Santini, S. Carretta, G. Amoretti, R. Caciuffo, N. Magnani, and G. H. Lander, Rev. Mod. Phys. 81, 807 (2009).

42 S. R. White, Nature Physics 8, 863 (2012); H.-C. Jiang, Z. Wang and L. Balents, Nature Phys. 8, 902 (2012); S. V. Isakov, R. G. Melko, and M. B. Hastings, Science 335, 193 (2012).

43 T.-H. Tan, J. S. Helton, S. Chu, D. G. Nocera, J. A. Rodriguez-Rivera, C. Broholm, and Y. S. Lee, Nature 492, 406 (2012). 